\title{
Effect of intermittent high intensity exercise on counter-regulatory hormones in type 1 diabetes glargine/glulisine users
}

\author{
Marie-Christine Dubé ${ }^{1,2}$, Carole Lavoie ${ }^{3}$ and S. John Weisnagel ${ }^{1,2^{*}}$ \\ *Correspondence: john.weisnagel@crchul.ulaval.ca

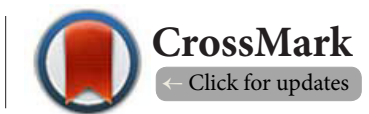 \\ 'Diabetes Research Unit, Université Laval Medical Center, Québec City, QC, Canada. \\ ${ }^{2}$ Molecular Endocrinology and Genomics, Université Laval Medical Center, Québec City, QC, Canada. \\ ${ }^{3}$ Department of Physical Activity Sciences, Université du Québec à Trois-Rivières, QC, Canada.
}

\begin{abstract}
Background: Brief intense sprint intervals during moderate intensity exercise prevent hypoglycemia in type 1 diabetic participants. We tested the hypothesis that this exercise modality would increase counter-regulatory hormones and limit BG decrease in glargine/glulisine insulin analogs users.

Findings: Participants performed, in a repeated measures, random-ordered design, 60 minutes of moderate intensity exercise at $50 \% \mathrm{VO}_{2 \text { peak }}$ in the following conditions: ingestion of $0 \mathrm{~g}$ of glucose pre-exercise, $30 \mathrm{~g}$ of glucose pre-exercise, or $0 \mathrm{~g}$ of glucose preexercise, but interspersed with brief high intensity intervals every 2 minutes. Intermittent brief high intensity exercise significantly increased levels of norepinephrine at mid-exercise (from $3.2 \pm 1.0$ to $7.8 \pm 3.2 \mathrm{nmol} / \mathrm{l}$ ) and cortisol from mid-exercise (from $330 \pm 159$ to $606 \pm 285 \mathrm{nmol} / \mathrm{l})$ to even after exercise $(692 \pm 334 \mathrm{nmol} / \mathrm{l})$ compared to other conditions.

Conclusions: Our results suggest that an intermittent high intensity exercise raised counter-regulatory hormones which may contribute to the prevention of exercise-induced hypoglycaemia in participants with type 1 diabetes using glargine/glulisine. Such exercise regimen can be an alternative to prevent exercise-induced hypoglycemia in glargine/glulisine users as seen in other insulin combinations.
\end{abstract}

Keywords: Hypoglycemia, glulisine, glargine, counter-regulatory hormones, intermittent high intensity exercise, strategies, type 1 diabetes

\section{Introduction}

Glulisine, a rapid acting insulin analog, may be useful in the treatment of allergy to rapid acting insulin and analogs [1] and can be an alternative to other analogs. It is highly probable that its effect on blood glucose (BG) and catecholamine would be similar to other insulin combinations but it needs to be documented when combined with glargine under various exercise conditions.

It has been shown that intermittent high-intensity exercise increased catecholamine and attenuated the decline in BG levels in type 1 diabetes mellitus (T1DM) under various insulin regimen [2] and reduced the risk of exercise-induced hypoglycemiain subjects using glargine/glulisine [3]. With the growing popularity of sports with this variable intensity profile such as many recreational team sports and some interval training, this exercise modality may be an interesting strategy to endorse for reducing the risk of exercise-induced hypoglycemia. The effect of different modalities of exercise intensity (moderate vs high) on counter-regulatory hormones needs to be documented in order to better prevent hypoglycaemia in physically active individuals with T1DM, a problem with major clinical impact.
Therefore, this study presents the effect of moderate $v s$ high intermittent intensity exercise i.e., 10 -seconds sprints every 2 minutes during 60-min exercise on counter-regulatory hormones in glargine/glulisine users.

\section{Methods}

Eleven moderately active participants with T1DM (five men and six women) participated in this study. They were free of diabetic complications and had no contra-indication for exercise. All participants were on the basal-bolus regimen using an insulin analogue glargine at bedtime and glulisine before every meal. This study was approved by the Ethic Committee of Centre Hospitalier Universitaire de Québec and all patients gave their informed written consent.

Participants were instructed not to engage in any preceding unusual or intense exercise on the days of exercise testing. Exercise tolerance was evaluated for each subject by using an incremental protocol of $15 \mathrm{~W} \cdot \mathrm{min}^{-1}$ (women) and $30 \mathrm{~W} \cdot \mathrm{min}^{-1}$ (men) after a warm-up period of 2 min performed on an electromagnetically braked cycle ergometer (Corival, Lode, The Netherlands) at a pedaling rate of 50 to $70 \mathrm{rpm}$. Participants 
Dube et al. Journal of Diabetes Research \& Clinical Metabolism 2014,

were given strong verbal encouragement to exercise to the highest tolerated symptoms of fatigue or dyspnea. Exercise was terminated at this point and/or when participants were unable to maintain speed or $\mathrm{Q} 40$ revolutions per minute on the ergo-cycle. Expired air was continuously recorded on a breath-by-breath basis for the determination of $\mathrm{vO}_{2^{\prime}}$ carbondioxide production $\left(\dot{\mathrm{V}} \mathrm{CO}_{2}\right), \dot{\mathrm{V}} \mathrm{E}$, and the RER $\left(\mathrm{VCO}_{2}\right)$ $\dot{\mathrm{V}}_{2}$ ) (CardiO2 CPX Ultima; Medical Graphics Co.). The HR was obtained from electrocardiographic monitoring. Blood pressure was measured every 2 min by using an automated sphygmomanometer with a headphone circuit option (Model 412; Quinton Instrument, Bothell, WA). $\dot{\mathrm{O}}_{2 \text { peak }}$ was defined as the mean $\mathrm{v}_{2}$ recorded in the last $15 \mathrm{~s}$ of the incremental exercise protocol concurrent withan RER of 1.15 or greater. Each participant performed 60 min of aerobic exercise on a cycle ergometer (Organic V3; Bodyguard Fitness, QC, Canada) at $50 \%$ of their previously determined $\dot{\mathrm{v}}_{2 \text { peak }}$ on two occasions and on one occasion with interspersed 10 -ss prints at maximal effort every 2 min of the exercise period.

The exercise conditions varied as follows: participants ingested a placebo snack of $0 \mathrm{~g}$ of carbohydrate $(\mathrm{CHO})$ preexercise i.e., sucaryl in $10 \%$ water solution (OG+MOD), $30 \mathrm{~g}$ of $\mathrm{CHO}$ pre-exercise (30G+MOD), or $0 \mathrm{~g}$ of $\mathrm{CHO}$ pre-exercise, but performed brief high intensity intervals interspersed every $2 \mathrm{~min}(0 \mathrm{G}+\mathrm{MOD} / \mathrm{INT})$ during the exercise trial. The $\mathrm{CHO}$ beverage consisted of $8 \mathrm{mg} \cdot \mathrm{kg}^{-1} \cdot \mathrm{min}^{-1}$ of dextrose in $10 \%$ water solution given $15 \mathrm{~min}$ before the beginning of exercise period. The exercise was scheduled $120 \mathrm{~min}$ after lunch and was followed by a 30 min-recovery period on site. To proceed to the experiments, no hypoglycemia was allowed 24 hours before testing. Moreover, subjects were requested to record their BG 24 hours following the exercise sessions and to report any hypoglycemia. All exercise tests were scheduled at least one week apart.

During the experimental protocols, a catheter (Cathlon Clear, Johnson \& Johnson, New Brunswick, NJ) was placedin an antecubital vein for sampling and kept patent by salinedrip. Blood samples were collected from time-15 min at 5- to 30min intervals for $2 \mathrm{~h}$ and then every 5 min duringand after the exercise period to measure BG concentrations by using a Freestyle glucometer (Abbott, California). Blood samples were centrifuged for plasma and stored at $-20^{\circ} \mathrm{C}$ for later analyses of glucose using a hexokinase method (13). Plasma catecholamines were measured by a method adapted from Raggi et al., [4]. Cortisol concentrations were measured by ECLIA (Roche Diagnostic GmbH, Mannheim, Germany). A glucose clamp procedure with a variable infusion of a $20 \%$ dextrose solution, adapted from DeFronzo et al., [5] was followed if needed, to avoid hypoglycemia. If at any time during the testing conditions glucose levels fell below 4 $\mathrm{mmol} / \mathrm{l}$, the dextrose infusion was initiated at a rate based on the drop in BG in order to maintain BG between 4 and 5 $\mathrm{mmol} / \mathrm{l}$. Intensity of exercise was estimated by the Borg scale of perceived exertion (6 to 20 scale) every 15 minutes. Area under the curve was calculated for $\mathrm{BG} \geq 8 \mathrm{mmol} / \mathrm{l}$ from the beginning of exercise until the end of the experiment. Values are expressed as means \pm SE. A multiple comparison procedure was performed using a Bonferroni correction to determine specific group differences with overall $P<0.016$ considered as significant using JMP 7 (SAS Institute, Cary, NC).

\section{Results}

Participants' characteristics aredescribed in Table 1. They were considered as low to moderately active. At arrival, BG was similar between conditions and became higher $5 \mathrm{~min}$ after starting the exercise until $30 \mathrm{~min}$ post-exercise in the $30 \mathrm{G}+\mathrm{MOD}$ condition compared to $\mathrm{OG}+\mathrm{MOD}$ and $\mathrm{OG}+\mathrm{MOD} /$ INT conditions, (all $P<0.02$ ), Figure 1. The first half of exercise induced a similar BG change between conditions of $-1.7 \pm 2.0$, $-1.4 \pm 1.2$ and $-1.1 \pm 1.3 \mathrm{mmol} / \mathrm{l}$, for the $0 \mathrm{G}+\mathrm{MOD}, 0 \mathrm{G}+\mathrm{MOD} /$

Table 1. Characteristics of the participants.

\begin{tabular}{lllll}
\hline & $\begin{array}{l}\text { All } \\
(\mathbf{n = 1 1 )}\end{array}$ & $\begin{array}{l}\text { Men } \\
(\mathbf{n = 5})\end{array}$ & $\begin{array}{l}\text { Women } \\
(\mathbf{n = 6})\end{array}$ & $\boldsymbol{P}$ value \\
\hline Variables & -- & -- & -- & -- \\
Age $(\mathrm{yrs})$ & $26.5 \pm 6.6$ & $27.6 \pm 8.0$ & $25.5 \pm 5.8$ & $\mathrm{NS}$ \\
$\mathrm{HbA}_{1 \mathrm{c}}(\%)$ & $7.3 \pm 0.4$ & $7.2 \pm 0.3$ & $7.4 \pm 0.5$ & $\mathrm{NS}$ \\
$\dot{\mathrm{VO}}_{\text {2peak }}\left(\mathrm{ml} \mathrm{O} \cdot \mathrm{kg}^{-1} \cdot \mathrm{min}^{-1}\right)$ & $33.4 \pm 6.5$ & $39.0 \pm 3.0$ & $28.7 \pm 4.4$ & 0.002 \\
Weight $(\mathrm{kg})$ & $74.1 \pm 6.7$ & $76.4 \pm 5.1$ & $72.3 \pm 7.7$ & $\mathrm{NS}$ \\
BMI $\left(\mathrm{kg} / \mathrm{m}^{2}\right)$ & $25.8 \pm 2.7$ & $25.0 \pm 3.1$ & $26.6 \pm 2.3$ & $\mathrm{NS}$ \\
Diabetes duration $(\mathrm{yrs})$ & $12.2 \pm 5.1$ & $12.4 \pm 5.8$ & $12.0 \pm 4.9$ & $\mathrm{NS}$ \\
Total daily dose $(\mathrm{U})$ & $58.7 \pm 22.7$ & $52.6 \pm 22.0$ & $63.8 \pm 25.3$ & $\mathrm{NS}$ \\
\hline
\end{tabular}

BMI: body mass index

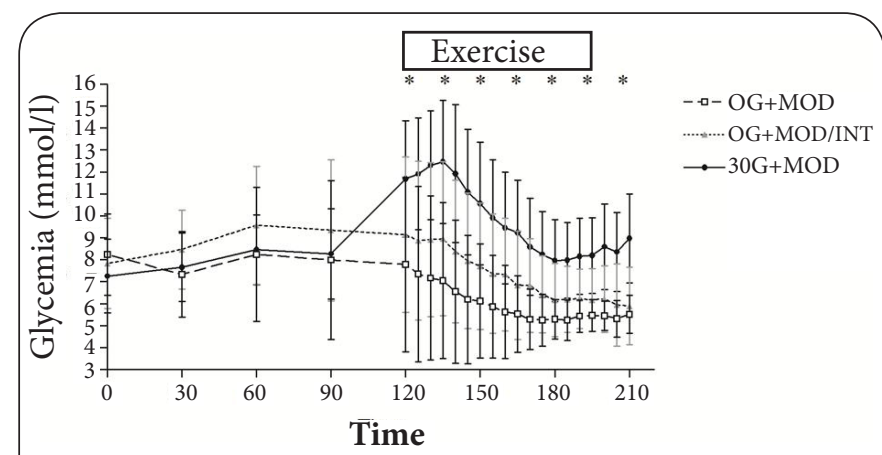

Figure 1. Glycemic excursions according to exercise conditions.

Open squares: 60 -min exercise session at $50 \% \mathrm{VO}_{2 \text { peak }}$ with a placebo beverage (OG+MOD); Grey triangles: 60-min exercise session at $50 \% \mathrm{VO}_{2 \text { peak }}$ interspersed with 10 -s maximal sprint effort every 2 minutes of the exercise period with a placebo beverage (OG+MOD/INT); Black circles: 60 -min exercise session at $50 \% \mathrm{VO}_{2 \text { peak }}$ with a $30 \mathrm{~g} \mathrm{CHO}$ beverage (30G+MOD) $P$ values reflect differences across the three conditions determined by ANOVA. A multiple comparison procedure was performed using a Bonferroni correction to determine specific group differences. Overall $\mathrm{P}$ values $<0.016$ are considered significant for time 125 to 210 minutes. 

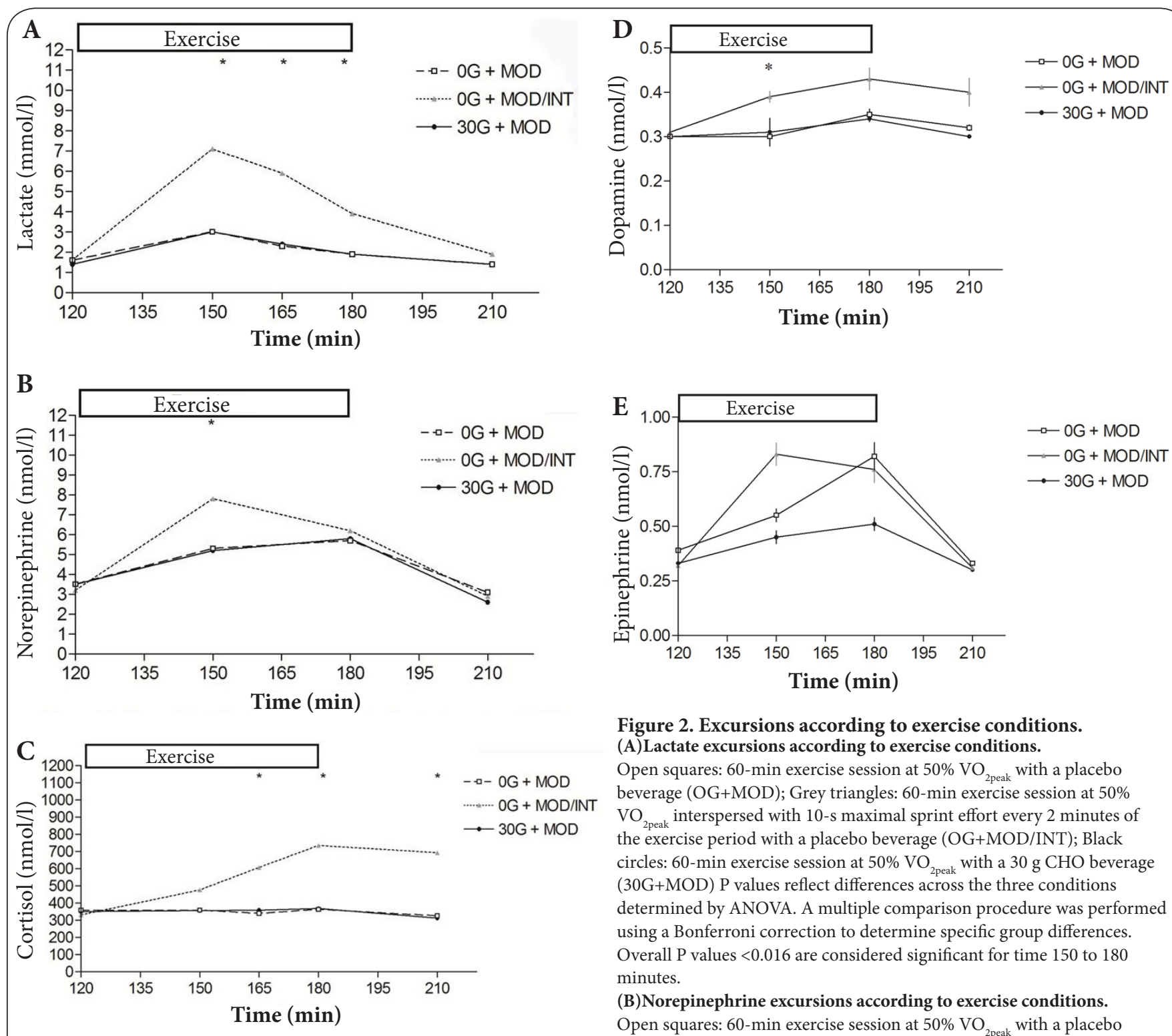

Figure 2. Excursions according to exercise conditions. (A)Lactate excursions according to exercise conditions. Open squares: 60 -min exercise session at $50 \% \mathrm{VO}_{2 \text { peak }}$ with a placebo beverage (OG+MOD); Grey triangles: 60 -min exercise session at $50 \%$ $\mathrm{VO}_{2 \text { peak }}$ interspersed with 10-s maximal sprint effort every 2 minutes of the exercise period with a placebo beverage (OG+MOD/INT); Black circles: 60 -min exercise session at $50 \% \mathrm{VO}_{2 \text { peak }}$ with a $30 \mathrm{~g} \mathrm{CHO}$ beverage (30G+MOD) P values reflect differences across the three conditions determined by ANOVA. A multiple comparison procedure was performed using a Bonferroni correction to determine specific group differences. Overall $\mathrm{P}$ values $<0.016$ are considered significant for time 150 to 180 minutes.

(B)Norepinephrine excursions according to exercise conditions. Open squares: 60 -min exercise session at $50 \% \mathrm{VO}_{2 \text { peak }}$ with a placebo beverage (OG+MOD); Grey triangles: 60 -min exercise session at $50 \% \mathrm{VO}_{2 \text { peak }}$ interspersed with 10-s maximal sprint effort every 2 minutes of the exercise period with a placebo beverage (OG+MOD/INT); Black circles: 60 -min exercise session at $50 \% \mathrm{VO}_{2 \text { peak }}$ with a $30 \mathrm{~g} \mathrm{CHO}$ beverage (30G+MOD) $\mathrm{P}$ values reflect differences across the three conditions determined by ANOVA. A multiple comparison procedure was performed using a Bonferroni correction to determine specific group differences. Overall $\mathrm{P}$ values $<0.016$ are considered significant at time $150 \mathrm{~min}$.

(C)Cortisol excursions according to exercise conditions.

Open squares: 60 -min exercise session at $50 \% \mathrm{VO}_{2 \text { peak }}$ with a placebo beverage $(\mathrm{OG}+\mathrm{MOD})$; Grey triangles: 60 -min exercise session at $50 \% \mathrm{VO}_{2 \text { peak }}$ interspersed with 10-s maximal sprint effort every 2 minutes of the exercise period with a placebo beverage (OG+MOD/INT); Black circles: 60-min exercise session at $50 \% \mathrm{VO}_{2 \text { peak }}$ with a $30 \mathrm{~g} \mathrm{CHO}$ beverage (30G+MOD) P values reflect differences across the three conditions determined by ANOVA. A multiple comparison procedure was performed using a Bonferroni correction to determine specific group differences. Overall $\mathrm{P}$ values $<0.016$ are considered significant for time 165 to 210 minutes.

(D)Dopamine excursions according to exercise conditions.

Open squares: 60-min exercise session at 50\% $\mathrm{VO}_{2 \text { peak }}$ with a placebo beverage (OG+MOD); Grey triangles: 60 -min exercise session at $50 \% \mathrm{VO}_{2 \text { peak }}$ interspersed with 10-s maximal sprint effort every 2 minutes of the exercise period with a placebo beverage (OG+MOD/INT); Black circles: 60-min exercise session at $50 \% \mathrm{VO}_{2 \text { peak }}$ with a $30 \mathrm{~g} \mathrm{CHO}$ beverage (30G+MOD) P values reflect differences across the three conditions determined by ANOVA. A multiple comparison procedure was performed using a Bonferroni correction to determine specific group differences. Overall $\mathrm{P}$ values $<0.016$ are considered significant for time 150 minutes.

(E)Epinephrine excursions according to exercise conditions.

Open squares: 60 -min exercise session at $50 \% \mathrm{VO}_{2 \text { peak }}$ with a placebo beverage (OG+MOD); Grey triangles: 60 -min exercise session at $50 \% \mathrm{VO}_{2 \text { peak }}$ interspersed with 10 -s maximal sprint effort every 2 minutes of the exercise period with a placebo beverage (OG+MOD/INT); Black circles: 60 -min exercise session at $50 \% \mathrm{VO}_{2 \text { peak }}$ with a $30 \mathrm{~g}$ CHO beverage (30G+MOD) P values reflect differences across the three conditions determined by ANOVA. A multiple comparison procedure was performed using a Bonferroni correction to determine specific group differences. 
Dube et al. Journal of Diabetes Research \& Clinical Metabolism 2014,

INT and 30G+MOD, respectively, $P=0.70$. The second half of exercise induced a mean $B G$ change of $-0.8 \pm 1.8,-1.5 \pm 1.6$ and $-2.6 \pm 1.4 \mathrm{mmol} / \mathrm{l}$, for $0 \mathrm{G}+\mathrm{MOD}, 0 \mathrm{G}+\mathrm{MOD} / \mathrm{INT}$ and $30 \mathrm{G}+\mathrm{MOD}$, respectively, $P=0.04$. This is mainly due to the fact that a higher number of subjects ( 7 of 11 ) in the $0 G+M O D$ group required dextrose infusion to prevent hypoglycemia $(P=0.05$ for group differences). Despite the infusion of dextrose in those subjects, four of them presented values slightly below $4 \mathrm{mmol} / \mathrm{l}$ although asymptomatic. Catecholamines measurements were compared between these 4 subjects and the others and no difference was shown. The area under the curve for $B G$ values $>8$ $\mathrm{mmol} / \mathrm{I}$ was higher in the $30 \mathrm{G}+\mathrm{MOD}$ condition compared to OG + MOD and $0 G+M O D / I N T$ conditions, (193 \pm 35 vs $46 \pm 28$ vs $102 \pm 45 \mathrm{mmol} / \mathrm{l} / \mathrm{min}, P<0.05)$, respectively. The $0 \mathrm{G}+\mathrm{MOD} / \mathrm{INT}$ condition showed higher values of lactate and norepinephrine at mid-exercise compared to the other exercise conditions, $P<0.05$, Figures $2 \mathrm{~A}$ and $\mathbf{2 B}$, respectively. The cortisol levels were higher in the $0 \mathrm{G}+\mathrm{MOD} / \mathrm{INT}$ condition after 45 minutes of exercise until 30 minutes post-exercise, all $P<0.005$, Figure $2 \mathrm{C}$. The dopamine levels at mid-exercise were higher in the OG+MOD/INT condition compared to $0 \mathrm{G}+\mathrm{MOD}$ and $30 \mathrm{G}+\mathrm{MOD}$ conditions, $P<0.01$, Figure 2D. The epinephrine levels tended to be higher in the $0 \mathrm{G}+\mathrm{MOD} / \mathrm{INT}$ condition compared to the other exercise conditions, $P=0.07$, Figure $2 \mathrm{E}$. Mean scores of perceived exertion were similar except at $15 \mathrm{~min}$ before the end of exercise $(10.9 \pm 2.0,13.8 \pm 2.4$ and $11.9 \pm 2.5)$ for the $0 \mathrm{G}+\mathrm{MOD}$ $0 \mathrm{G}+\mathrm{MOD} / \mathrm{INT}$ and $30 \mathrm{G}+\mathrm{MOD}$ conditions, respectively, $P<0.05$. The rate of late-onset exercise-induced hypoglycemia was recorded and there was no difference $(0.27 \pm 0.14,0.33 \pm 0.17$ and $0.20 \pm 0.13$ events) for the $0 \mathrm{G}+\mathrm{MOD}, 0 \mathrm{G}+\mathrm{MOD} / \mathrm{INT}$ and $30 \mathrm{G}+\mathrm{MOD}$ conditions, respectively.

\section{Discussion}

Intermittent high intensity exercise reduced the decline in BG during exercise in participants with T1DM $[2,6]$. Repeated intense sprints interrupting longer periods of moderateintensity exercise is typical of interval training which has gained in popularity for its favorable impact on cardiovascular function [7] and may simulate the type of exercise performed in many sports.

Our results show that intermittent high intensity $60 \mathrm{~min}-$ exercise sessions raised counter-regulatory hormones during and following exercise in glargine/glulisine T1DM users. Glulisine may be useful in the treatment of allergy to rapid acting insulin and analogs [1], and can be an alternative to other rapid-acting analogs. Therefore, in this regimen, we specifically showed that norepinephrine, dopamine and cortisol levels were significantly higher while epinephrine tended to be higher in the $0 G+M O D / I N T$ condition. Exerciseinduced hypoglycemia was found in 7 subjects in the $0 \mathrm{G}+\mathrm{MOD}$ condition compared to only 1 in the $0 G+M O D / I N T$ condition, requiring the start of a dextrose infusion. Despite this infusion, four subjects still presented bordeline glycemic values with BG between 3.6 and $4 \mathrm{mmol} / \mathrm{l}$. However, catecholamine were compared between the «bordeline-hypoglycemic subjects» and the others and no differences were seen in any of the measures suggesting that higher catecholamines values in the OG+MOD/INT condition were attributable to exercise. These increases were more pronounced around mid-exercise and after, at a period when the Borg scale was significantly elevated as well. Lactate levels were also raised, especially from midto end of exercise. Elevated lactate levels contribute to the stabilisation of BG by providing gluconeogenic precursors for hepatic glucose production [8], notably at the onset of recovery from moderate-intensity exercise interspersed either with several short sprints [9] or followed by a 10-s sprint [10]. Iscoe et al., [11] observed similar differences in counter-regulatory hormones between continuous moderate-intensity exercise and continuous moderate-intensity + intermittent highintensity exercise, which was explained by a modest increase in sympathetic counter-regulatory response resulting from the performance of a heavier resistance-type exercise rather than a faster cycling cadence [12].

The reduced risk of early-onset hypoglycemia applying with a high intensity intermittent exercise was not accompanied by a higher risk of late-onset exercise-induced hypoglycemia as indicated by measured BG for 24 hours.

Although beneficial to prevent hypoglycemia, the $0 \mathrm{G}+\mathrm{MOD} /$ INT condition was harder to perform compared to moderate intensity exercise conditions as reflected by the Borg scale scores. Therefore, intermittent high intensity exercise may constitute a strategic choice to prevent exercise-induced hypoglycemia but requires a certain level of fitness and tolerance to exercise exertion. However, it remains an interesting option in individuals who have not modified their pre-exercise insulin doses or prefer to avoid extra $\mathrm{CHO}$. In addition, differential responses of BG levels and counter regulatory hormones to varying exercise conditions reflects the need to precisely take into account the type of exercise performed by the individual with T1DM in the management of the disease and preventive of exercise-induced hypoglycemia.

\section{Competing interests}

The authors declare that they have no competing interests.

Authors' contributions

\begin{tabular}{|l|c|c|c|}
\hline Authors' contributions & MD & CL & SW \\
\hline Research concept and design & $\checkmark$ & $\checkmark$ & $\checkmark$ \\
\hline Collection and/or assembly of data & $\checkmark$ & -- & -- \\
\hline Data analysis and interpretation & $\checkmark$ & -- & $\checkmark$ \\
\hline Writing the article & $\checkmark$ & -- & -- \\
\hline Critical revision of the article & $\checkmark$ & $\checkmark$ & $\checkmark$ \\
\hline Final approval of article & $\checkmark$ & $\checkmark$ & $\checkmark$ \\
\hline Statistical analysis & $\checkmark$ & -- & -- \\
\hline
\end{tabular}

Acknowledgment and funding

We would like to thank all participants and the personnel of the Diabetes Research Unit of the CHUL. This study 
was supported by Sanofi aventis Canada inc.

\section{Publication history}

EIC: Geoffrey Burnstock, University College London, UK.

Received: 17-Apr-2014 Final Revised: 20-May-2014

Accepted: 09-Jun-2014 Published: 25-Jun-2014

\section{References}

1. Mollar-Puchades MA and Villanueva IL. Insulin glulisine in the treatment of allergy to rapid acting insulin and its rapid acting analogs. Diabetes Res Clin Pract. 2009; 83:e21-2. | Article | PubMed

2. Guelfi KJ, Ratnam N, Smythe GA, Jones TW and Fournier PA. Effect of intermittent high-intensity compared with continuous moderate exercise on glucose production and utilization in individuals with type 1 diabetes. Am J Physiol Endocrinol Metab. 2007; 292:E865-70. | Article PubMed

3. Dube MC, Lavoie $C$ and Weisnagel SJ. Glucose or intermittent highintensity exercise in glargine/glulisine users with T1DM. Med Sci Sports Exerc. 2013; 45:3-7. | Article | PubMed

4. Raggi MA, Sabbioni C, Casamenti G, Gerra G, Calonghi N and Masotti L. Determination of catecholamines in human plasma by highperformance liquid chromatography with electrochemical detection. J Chromatogr B Biomed Sci Appl. 1999; 730:201-11. | Article | PubMed

5. DeFronzo RA, Tobin JD and Andres R. Glucose clamp technique: a method for quantifying insulin secretion and resistance. Am J Physiol. 1979; 237:E214-23. | Pdf | PubMed

6. Galassetti PR, Iwanaga K, Crisostomo M, Zaldivar FP, Larson J and Pescatello A. Inflammatory cytokine, growth factor and counterregulatory responses to exercise in children with type 1 diabetes and healthy controls. Pediatr Diabetes. 2006; 7:16-24. | Article | PubMed

7. Trilk JL, Singhal A, Bigelman KA and Cureton KJ. Effect of sprint interval training on circulatory function during exercise in sedentary, overweight/obese women. Eur J Appl Physiol. 2011; 111:1591-7. Article | PubMed

8. Vettor R, Lombardi AM, Fabris R, Pagano C, Cusin I, Rohner-Jeanrenaud $\mathrm{F}$, Federspil $\mathrm{G}$ and Jeanrenaud $\mathrm{B}$. Lactate infusion in anesthetized rats produces insulin resistance in heart and skeletal muscles. Metabolism. 1997; 46:684-90. | Article | PubMed

9. Guelfi KJ, Jones TW and Fournier PA. The decline in blood glucose levels is less with intermittent high-intensity compared with moderate exercise in individuals with type 1 diabetes. Diabetes Care. 2005; 28:1289-94. | Article | PubMed

10. Bussau VA, Ferreira LD, Jones TW and Fournier PA. The 10-s maximal sprint: a novel approach to counter an exercise-mediated fall in glycemia in individuals with type 1 diabetes. Diabetes Care. 2006; 29:601-6. | Article | PubMed

11. Iscoe KE and Riddell MC. Continuous moderate-intensity exercise with or without intermittent high-intensity work: effects on acute and late glycaemia in athletes with Type 1 diabetes mellitus. Diabet Med. 2011 28:824-32. | Article | PubMed

12. Maran A, Pavan P, Bonsembiante B, Brugin E, Ermolao A, Avogaro A and Zaccaria $\mathrm{M}$. Continuous glucose monitoring reveals delayed nocturnal hypoglycemia after intermittent high-intensity exercise in nontrained patients with type 1 diabetes. Diabetes Technol Ther. 2010; 12:763-8. | Article | PubMed

\section{Citation:}

Dubé M-C, Lavoie C and Weisnagel SJ. Effect of intermittent high intensity exercise on counter-regulatory hormones in type 1 diabetes glargine/glulisine users. $J$ Diab Res Clin Met. 2014; 3:8.

http://dx.doi.org/10.7243/2050-0866-3-8 